\title{
Application of Human Pluripotent Stem Cells in T Cell Based Cancer Immunotherapy.
}

\author{
Arvind Chhabra * \\ Department of Medicine, University of Connecticut Health Center, Farmington, Connecticut, USA. \\ * Author to whom correspondence should be addressed; Department of Medicine, University of \\ Connecticut Health Center, Farmington, Connecticut, USA-06030; Tel.: 860-679-1447; E-Mail: \\ arvindac@yahoo.com
}

Received: 18 August 2011 / Accepted: 23 September 2011 / Published: 28 September 2011

\begin{abstract}
Human pluripotent stem cells represent a unique reservoir that could be utilized for generating cells of a given lineage. Cancer immunotherapy field could benefit from this, since one of the key limitations in developing an effective immune based cancer therapy is the lack of sufficient high avidity anti-tumor $\mathrm{T}$ cells in a great majority of cancer patients. The main reason for this is the fact that most human tumor antigens are "self-antigens" and most self reactive high avidity $\mathrm{T}$ cells are deleted during development to avoid autoimmunity. This manuscript reviews recent advances towards differentiating human pluripotent stem (hPS) cells into different immune effector cells, and potential strategies to utilize hPS cells in $\mathrm{T}$ cell based cancer immunotherapy approaches. We will also discuss the advantages and concerns associated with their clinical applications.
\end{abstract}

Keywords: Cancer; Immunotherapy; TCR engineering; Human pluripotent stem cells (hPS); Human embryonic stem cells (hES); induced pluripotent stem cells (iPS).

Commonly Used Abbreviations: CTL: Cytolytic T Lymphocytes, APC: Antigen Presenting Cells, TCR: T Cell Receptor, hPS: Human Pluripotent Stem Cells, hES: Human Embryonic Stem Cells, iPS: Induced Pluripotent Stem Cells, CRT: Cell Replacement Therapy. 


\section{Introduction:}

The development of our immune system is programmed in such a way that we have a complex network of immune effectors that work in a coherent fashion to respond to the invading infectious agents and protect us. Although the specificity and protective efficacy of immune effectors to target "foreign agents" has been established elegantly, it has long been quite a difficult puzzle for the tumor immunologists to understand why the host immune system cannot mount a productive anti-tumor immune response to target cancer cells and prevent it from developing into a tumor mass.

A careful analysis of the tumor development process suggests that the normal host cellular architecture, in response to several defined as well as yet undefined chain of events, transforms to give rise to a small cell population, now termed as cancer stem cell [1], that leads to the development of the tumor mass. Since cancer arise from within, i.e. cancer cells display a "self" cellular architecture, host immune mechanisms cannot recognize and target them as efficiently as they would do so against the foreign infectious agents and the host cells infected with pathogens. As a result, a growing tumor goes undetected. Although, it is understandable that the nature would not allow the host immune system to target its "self" cellular architecture, as this would lead to autoimmunity, it has long been the goal of tumor immunologists to utilize the specificity and efficacy of host immune system to effectively target and eradicate the tumor.

Despite numerous hurdles and countless failures, remarkable clinical responses observed in a limited number of cancer patients, whether it is through the active specific immunization based approaches or through adoptive administration of ex-vivo expanded anti-tumor $\mathrm{T}$ cells, have clearly established that the $\mathrm{T}$ cell based cancer immunotherapy can produce an effective clinical response, with far less side-effects that the chemotherapy or radiotherapy [2-9]. However, overall clinical outcome has not yet matched their potential or the expectations [10,11]. Novel strategies are needed to harness the therapeutic potential of immune based cancer therapy approaches for the greater public good. Interestingly, recent advances in human pluripotent stem cell field offer a unique opportunity to tumor immunologists, not only to address the issues of existence and the biology of "cancer stem cells", but also to utilize the human pluripotent stem cells (hPS) to generate immunologically matched, patient specific, immune effectors, in sufficiently large numbers and with desired immunological properties, to effectively target the tumor mass. This manuscript provides a brief overview of the $\mathrm{T}$ cell based cancer immunotherapy approaches, recent advances in human embryonic stem cells (hES) field, progress towards generating donor specific induced pluripotent stem cell (iPS) lines, derivation of immune effectors from hPS (hES and iPS) cells, and its potential significance for the cancer immunotherapy field.

\section{T Cell Based Cancer Immunotherapy}

While foreign infectious agents are dealt with by the antibody-mediated humoral arm of the host immune system, T cell-mediated cellular immunity is critical for targeting the transformed cells as well as the cells infected with foreign pathogens, displaying an altered cellular architecture than the normal host cells. T cells can be put into two broad categories, CD4+ T cells and the CD8+ T cells. During developmental cascade, hematopoietic stem cells (HSC) give rise to $\mathrm{CD} 4+\mathrm{CD} 8+$ double positive $\mathrm{T}$ lineage progenitors that lead to the development of single positive CD4 and CD8 T cells. Mature CD4 
and CD8 effector $\mathrm{T}$ cells recognize their target in context to specific MHC molecules and perform distinct roles to shape up the host $\mathrm{T}$ cell immunity. CD8 $\mathrm{T}$ cells recognize their targets in context to MHC class I molecules, exhibit antigen specific cytolytic effector response and are categorized as the "lytic effectors". CD4 T cells on the other hand recognize their target antigenic epitope in context to MHC class II molecules and can contribute as the immune facilitators/helpers or as the immune suppressors/regulators. While most early active specific as well as adoptive cancer immunotherapy approaches were aimed at generating CD8+ anti-tumor cytolytic T lymphocyte (CTL) response, it is now well recognized that CD4 play essential role in mounting a productive CD8+ CTL response [12], and incorporating CD4 $\mathrm{T}$ cells in cancer immunotherapy protocols could produce impressive clinical results $[13,14]$.

Interestingly, a growing tumor employs multiple strategies to escape protective immune response [15]. Among these includes the creation of a complex immunosuppressive tumor microenvironment that leads to immune dysfunction of functional anti-tumor immune effectors and induction of CD4+ suppressor/regulatory $\mathrm{T}$ cells. Concerted efforts are underway to improve the efficacy of active specific immunization as well as adoptive immunotherapy based cancer immunotherapy approaches through multiple strategies $[10,16]$. Among these includes, improving the immunogenic potential of antigen presenting cells (APC) [17], utilizing novel antigen delivery tools to facilitate an optimum processing and presentation of the antigen by the APC to the T cell precursors $[18,19]$, mitigating the negative signals causing dysfunction of functional anti-tumor $\mathrm{T}$ cells [20], and also by interfering with the activation induced cell death (AICD) mediated premature elimination of tumor reactive CTL [21, $22]$ to facilitate the generation of a robust and long lasting anti-tumor $T$ cell response.

Although these approaches have sufficient merit, as mentioned before, since cancer cells have "self" cellular architecture and most human tumor associated antigens are "self antigens", immune repertoire of most cancer patients does not harbor sufficient high avidity anti-tumor $\mathrm{T}$ cell precursors to mount a protective anti-tumor immune response. To address this issue, methods are being developed to genetically engineer donor derived normal $\mathrm{T}$ cell populations to function as potent anti-tumor effectors. Among these approaches includes, by engrafting human peripheral blood derived $\mathrm{T}$ cells with a transgenic TCR derived from anti-tumor $\mathrm{T}$ cells of cancer patients that harbor high avidity tumor antigen specific $\mathrm{T}$ cells or by engrafting them with the hybrid antibody- $\mathrm{T}$ cell molecules, termed T-body, comprised of the antigen recognition domain of an antibody and the signal transduction domain of a $\mathrm{T}$ cell receptor [23-25].

TCR engineered anti-tumor $\mathrm{T}$ cells have been shown to produce significant clinical response in melanoma patients [2]. However, several concerns have been raised against the clinical use of mature effector $\mathrm{T}$ cells, engineered with either approach, since mature $\mathrm{T}$ cells already possess TCR of their primary antigenic epitope specificities [24]. This could not only limit the transduction efficiency of the transgenic TCR, novel chimeric hybrid TCRs of undesired reactivity might also be generated with harmful consequences. In this context, the availability of methods to derive immune effectors from iPS cells would make it is feasible to create a sufficiently large reservoir of patient specific anti-tumor $\mathrm{T}$ cells through TCR engineering approach, that would not only harbor a defined tumor antigen specific transgenic TCR, their functional properties could also be customized, to effectively target the growing tumor. 


\section{Recent Advances In Human Pluripotent Stem Cell Research}

Human pluripotent stem cells (hPS) represent a unique, homogenous, pluripotent cell population that can be indefinitely maintained in an undifferentiated state under controlled culture conditions, and can be differentiated into desired cell lineages under appropriate differentiation conditions. Although, the therapeutic potential of the hPS based cell replacement therapy (CRT) is immense, until recently, embryonic stem cells were considered as the sole source of pluripotent cells that could be utilized to derive cell lineage(/s) of choice. The ethical and moral issues associated with the utilization of human embryos to derive embryonic stem cell lines [26] have long been a major roadblock in advancing the embryonic stem cell research and harnessing the therapeutic potential of hES cells, in a socially responsible manner. In addition, since hES lines derived from a different donor would be allogenic to the recipient's immune system, i.e. these cells would be recognized as "foreign", potential immune rejection of hES derived cells used for CRT presents an additional barrier towards a therapeutic use of hES derived cells.

Several approaches are being developed to address the potential immune rejection of hES derived cells. Among these includes somatic cell nuclear transfer (SCNT) approach [27] and the development of a sufficiently large hES bank, genetic modification of hES cells etc. In SCNT approach, patient's somatic cell derived nuclei is introduced into an enucleated donor oocyte to derive cloned embryos [27], from which patient matches hES lines can be derived. Creation of a large hES bank has been proposed to ensure the availability of HLA matched hES cells for CRT. In addition, genetic modification of hES cells making them immune receptive and the administration of immune modulators, such as mesenchymal stem cells, before hES based CRT have also been proposed. Although technically feasible, these approach do not address the ethical and moral issues associated with hES based therapeutics to advance them into clinic.

Interestingly, following the observations that the fusion of hES and somatic cells lead to dedifferentation of somatic cell nuclei [28], key re-programming factors were identified that could be utilized to generate iPS cells from the somatic cells [29, 30]. While initial reports on iPS generation utilized recombinant viral vectors to deliver reprogramming factors, several different methodologies are now available to generate iPS cells by non-viral methods [31], addressing concerns associated with the clinical application of recombinant viral vectors. The availability of the technical know-how to derive iPS cells offer a unique opportunity not only to study the hPS cells without any moral or ethical constraints, the development of donor specific iPS lines as the source material for the cell replacement therapy (CRT) would also address the issue of immune rejection associated with allogenic hES derived immune-incompetent cells, therefore, making it feasible to harness the therapeutic potential of hPS cells. Figure. 1 schematically shows the methodology to generate iPS lines from human somatic cells, and the iPS based CRT. 
Figure. 1. Schematic representation of derivation of patient specific induced pluripotent stem cell (iPS) lines and patient specific cell replacement therapy (CRT).

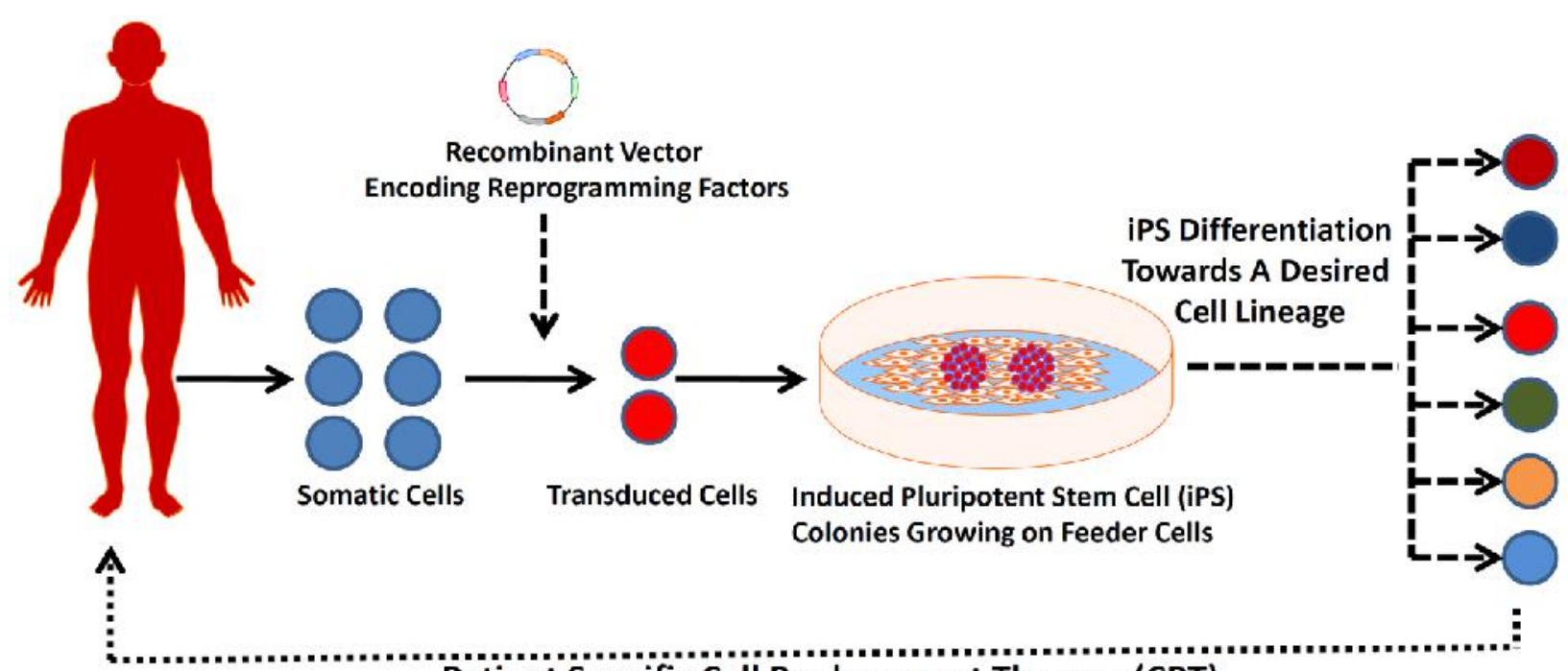

Patient Specific Cell Replacement Therapy (CRT)

While these developments are encouraging, concerns have also been raised about the safety and therapeutic potential of iPS cells, since many human cancers have been shown to have up-regulated expression levels of reprogramming factors used to derive iPS cells from somatic cells [32-34]. Systematic studies are needed to fully understand the level of similarities/ differences between iPS and hES lines, establish the relative efficacy of iPS cells to generate different cell lineages and their detailed functional characterization, in comparison to hES derived cells. In addition, the tumorigenic potential of iPS derived cells, in comparison to the hES derived cells, also needs to be carefully examined.

\section{Progress Towards Generating hematopoietic progenitors and Immune Effectors From Human Pluripotent Stem Cells}

All the blood cell lineages are derived from a common progenitor, the hematopoietic stem cells (HSC), through a progressive differentiation process termed hematopoiesis. HSC are characterized by their ability to differentiate into different hematopoietic lineages while retaining their capacity to selfrenew [35], as well as by an extensive proliferation capacity that decreases during ontogeny [36]. The HSC compartment is a heterogeneous pool separated into several distinct subpopulations based on both surface marker expression and retrospective identification of HSC, using in vivo and in vitro stem cell assays. Following the generation of HSC, the differentiation cascade of HSC follows two distinct paths, leading to the generation of lymphoid and myeloid progenitors. While lymphoid lineage progenitors lead to the development of T, B, and NK cells, the myeloid lineage progenitors lead to the generation of monocytes, granulocytes, erythrocytes and platelets.

In human fetal liver, umbilical cord blood (CB), and the bone marrow, $0.5 \%-5 \%$ of hematopoietic cells express CD34 cell surface marker [37], and the cells with this phenotype harbor virtually all in vitro clonogenic potential [38]. The adult CD34+ hematopoietic precursors have been characterized in details and methodologies have also been developed to differentiate the human bone marrow and/ or 
cord blood derived adult CD34+ cells into APC [39] and T cells [40-42]. Interestingly, the pool of CD34+ human hematopoietic stem cells is heterogeneous, and among the CD34+ cells, cells that do not express mature lineage marker, $\mathrm{CD} 38$, i.e. $\mathrm{CD} 34^{+} \mathrm{CD} 38^{-}$cells, are regarded as the long-term culture-initiating cells [35]. Over the last few years, a significant progress has been made towards the differentiation of hPS (hES \& iPS) cells into different cell types, including HSC [43-49], and the cell surface markers, CD34, CD38, CD45, KDR etc., have been used to track the generation of HSC [45, 48, 50, 51]. While CD34 antigen has long been considered as the key positive marker for human hematopoietic stem and progenitor cells $[35,52]$, and most studies on differentiation of hES cells into HSC have also relied on CD34 as the key marker to track the development of hematopoietic precursors [45, 49-51], it is now well established that the human HSC can be CD34+ve as well as CD34-ve [53].

The availability of a reliable method to generate functional HSC from hES would offer a large reservoir of HSC to derive different types of patient matched immune effectors. Towards this, methodologies have been developed to differentiate the hPS (hES/ iPS) derived CD34+ cells into natural killer (NK) cells [49], T cells [45, 51], and antigen presenting cells (APC) [50]. Although these reports are encouraging, development of methods for an optimal expansion of hPS derived HSC, their differentiation into different cell types, and a detailed functional characterization of HSC derived cell types are essential to advance them to the clinic.

\section{Strategies to Utilize Human Pluripotent Stem Cells In T Cell Based Cancer Immunotherapy Approaches}

As discussed before, most $\mathrm{T}$ cell based cancer immunotherapy approaches, whether it active specific immunization or the adoptive immunotherapy based approaches, utilize three key immune effectors, the APC, CD8 T cells and CD4 T cells. The development of methods to identify, characterize and isolate human monocytes, differentiate them into professional APCs, and maintain such APC in culture conditions, led to the development of several active specific immunization approaches. These approaches utilized administration of APCs, either pulsed with short synthetic peptides representing human tumor associated antigenic epitope(/s) or genetically modified, recombinant viral or non-viral vectors expressing human tumor antigen(/s) to effectively process and present $\mathrm{T}$ cell epitopes to the immune repertoire of cancer patients, with an objective to generate productive anti-tumor $\mathrm{T}$ cell responses. It should be noted that human peripheral blood derived APC can be immunogenic as well as tolerogenic, and one of the key factor determining the success of APC based active specific immunization schema is the immunogenic potential of APC. Several approaches are being pursued to augment the quality of the APC, either by modifying them by introducing immune facilitator cytokines, such as GM-CSF, or by knocking down endogenous immune suppressor agents.

With the development of methods to generate patient specific APC and CD4 and CD8 T cells from patient derived iPS cells, cancer immunotherapy field can enormously benefit from these advances. Figure. 2 schematically shows some of the potential approaches to utilize human pluripotent stem cells in cancer immunotherapy. As shown it is conceptually possible to derive patient specific APC with defined immunogenic properties from patient derived iPS cells that would produce a superior clinical outcome, as the quality of such APC could be better controlled, in the absence of the host 
immune suppression mechanisms. Towards this, recent progress towards generating antigen presenting cells from hPS cells in several different laboratories is quite encouraging [54-57].

Figure. 2. Potential strategies to utilize human pluripotent stem cells in cancer immunotherapy.

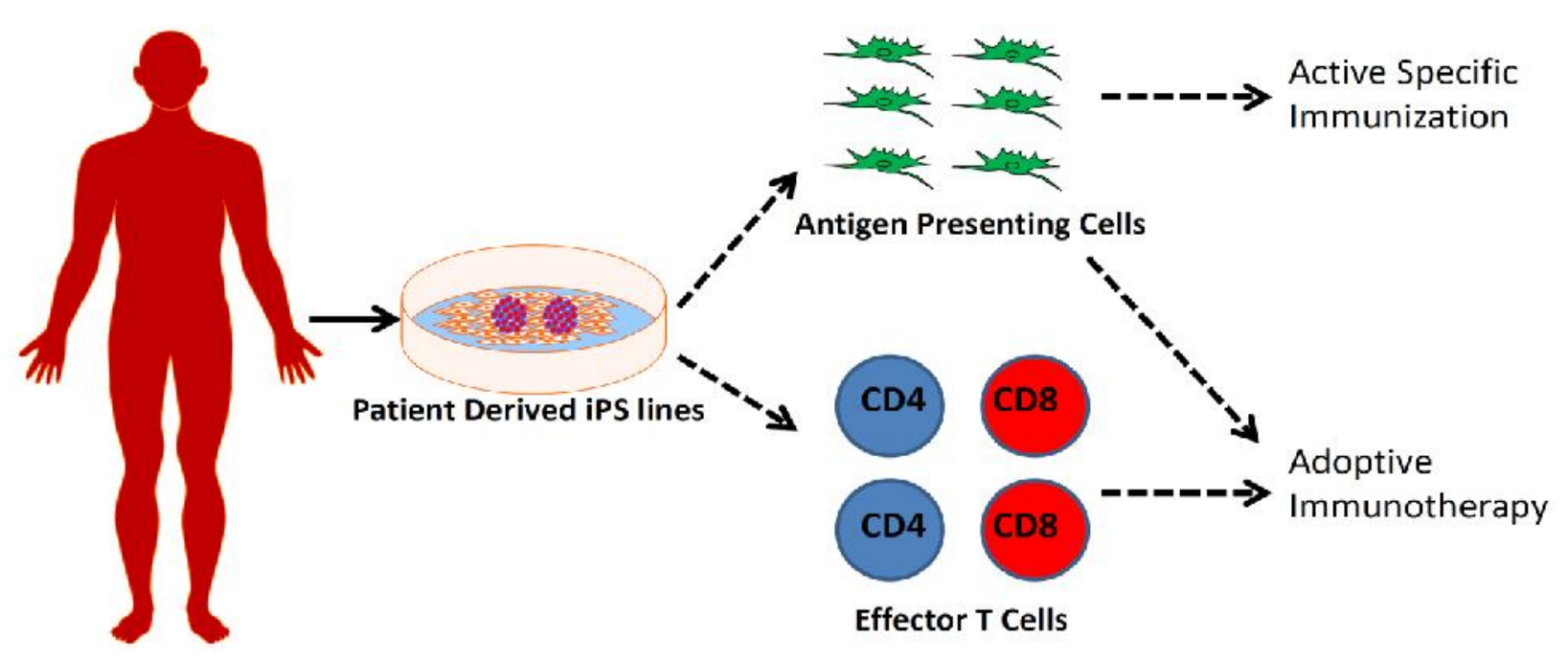

As mentioned before, extremely low frequency of high avidity anti-tumor T cell precursors in most cancer patients and the lack of an efficient method to simultaneously engage CD4 and CD8 T cells in tumor immunity, especially in an antigen specific manner, represent key limitations towards developing an effective cancer immunotherapy. Interestingly, we have recently shown that the TCR engineering approach can not only be utilized to generate customized anti-tumor effector T cells; with the availability of an appropriate MHC class I restricted transgenic TCR, it is also possible to engage human CD4 T cells in tumor immunity, as MHC class I directed "helper as well as lytic effectors" $[58,59]$. While these findings have significant clinical implications, concerns have been raised towards the clinical utilization of the transgenic TCR engineered mature effector T cells, since mature $\mathrm{T}$ cells possess TCR of their primary functional specificities. It is possible that the introduction of transgenic TCR might lead to TCR-mixing, resulting in creation of novel chimeric TCRs with undefined functional reactivities as well as self reactivities, in TCR engineered T cells. Recent findings in animal model showing fatal graft versus host reaction [60] emphasize the need to establish strict quality control checkpoints for clinical application of the engineered anti-tumor T cells. In this context, with the availability of methods to derive effector T cells from hPS cells it is conceptually possible to create an enormous reservoir of patient specific anti-tumor T cells that would express the TCR of a desired functional specificity, through TCR engineering approach. In addition to the above discussed approaches, HSC administration has been shown to produce an additive effect in animal model [61]. Anti-tumor potential of NK cells is also well established. Therefore, HSC and NK cells derived from hPS cells could also be utilized in cancer immunotherapy.

Interestingly, a recent study in mouse model has demonstrated anti-tumor effect of hES cells [62], in support of the long standing view that the administration of embryonic materials can induce antitumor effect [63]. Although these findings are encouraging, the prevailing ethical and moral issues associated with the derivation of HLA matched embryonic material and the tumorigenic potential of 
the hPS cells makes the therapeutic application of hPS cells as anti-cancer immunogenic agents a challenge. While the HLA gene engineered hES cells could be utilized to derive recipient HLA matched cells [64], patient derived iPS generated immune effectors might be the best clinical route. With the availability of methods to differentiate hPS cells into HSC [47-49], HSC into APC [39, 50], NK cells [49], as well as effector T cells [40-42, 45, 51], systematic studies are needed to examine the functional properties of the hPS derived immune effectors, especially in context to a physiologically relevant human tumor associated antigen. Studies in animal models [65] will also be critical to establish the in-vivo anti-tumor potential of the hPS derived immune effectors, in order to advance them to the clinic.

\section{Conclusion}

Among the key developments that could be credited to bring the human pluripotent stem cell research field to its present status includes, derivation of human embryonic stem cell lines [26], the creation of Dolly the sheep through SCNT technology [27], observations that the fusion of hES cells with somatic cells results in de-differentiation of the somatic cell nucleus [28], identification of key reprogramming factors that could be utilized to derive iPS cells from somatic cells [29, 30], etc. Although the prevailing belief that iPS cells are similar to the hES cells and recent progress towards deriving different cell types from iPS cells offer hope that the hPS based CRT would just not remain a distant goal but would soon become a reality, however, several critical issues need to be addressed to advance iPS derived cells to the clinic. Among these includes, establishing the level of similarity between iPS and hES cells, development of optimum differentiation conditions to derive cell lineages of choice from iPS cells, detailed functional characterization of iPS derived cell lineages etc. Understanding the tumorigenic potential of iPS derived cells is also critical since reprogramming factors used to induce iPS generation have been shown to be up-regulated in various cancer cells. In addition, key quality control checkpoints needs to be developed to ensure that the undifferentiated iPS cells are not administered to the recipient that could lead to tumor development. With the availability of a detailed understanding of the biology, safety and efficacy of iPS derived cells along these lines, iPS cells could very well turn out to be a key asset for the treatment of various debilitating diseases, including cancer.

\section{Acknowledgements}

Author thanks Dr. Bijay Mukherji for critical reading of the manuscript and helpful suggestions. Author also thanks all the contributors of the studies that have been cited here and many more whose elegant works have helped advance the field but could not be cited here merely due to the space limitations. Grant support from the State of Connecticut Stem Cell Research Initiative, and the Breast Cancer Alliance, Greenwich, Connecticut are also duly acknowledged. 


\section{References}

1. Scatena, R., et al., Cancer stem cells: the development of new cancer therapeutics. Expert opinion on biological therapy 2011, 11(7): 875-92.

2. Morgan, R.A., et al., Cancer regression in patients after transfer of genetically engineered lymphocytes. Science 2006, 314(5796): 126-9.

3. Dudley, M.E., et al., Cancer regression and autoimmunity in patients after clonal repopulation with antitumor lymphocytes. Science 2002, 298(5594): 850-4.

4. Hsu, F.J., et al., Vaccination of patients with B-cell lymphoma using autologous antigen-pulsed dendritic cells. Nat Med 1996, 2(1): 52-8.

5. Mukherji, B., et al., Induction of antigen-specific cytolytic T cells in situ in human melanoma by immunization with synthetic peptide-pulsed autologous antigen presenting cells. Proc Natl Acad Sci U S A 1995, 92(17): 8078-82.

6. Marchand, M., et al., Tumor regressions observed in patients with metastatic melanoma treated with an antigenic peptide encoded by gene MAGE-3 and presented by HLA-A1. Int $J$ Cancer 1999, 80(2): 219-30.

7. Thurner, B., et al., Vaccination with mage-3A1 peptide-pulsed mature, monocyte-derived dendritic cells expands specific cytotoxic $\mathrm{T}$ cells and induces regression of some metastases in advanced stage IV melanoma. J Exp Med 1999, 190(11): 1669-78.

8. Rosenberg, S.A. and M.E. Dudley, Adoptive cell therapy for the treatment of patients with metastatic melanoma. Current opinion in immunology 2009, 21(2): 233-40.

9. Rosenberg, S.A., M.E. Dudley, and N.P. Restifo, Cancer immunotherapy. The New England Journal of Medicine 2008, 359(10): 1072.

10. Rosenberg, S.A., J.C. Yang, and N.P. Restifo, Cancer immunotherapy: moving beyond current vaccines. Nat Med 2004, 10(9): 909-15.

11. Rosenberg, S.A., et al., Immunologic and therapeutic evaluation of a synthetic peptide vaccine for the treatment of patients with metastatic melanoma. Nat Med 1998, 4(3): 321-7.

12. Schoenberger, S.P., et al., T-cell help for cytotoxic T lymphocytes is mediated by CD40-CD40L interactions. Nature 1998, 393(6684): 480-3.

13. Chhabra, A., MHC class I TCR engineered anti-tumor CD4 T cells: implications for cancer immunotherapy. Endocrine, metabolic \& immune disorders drug targets 2009, 9(4): 344-52.

14. Hunder, N.N., et al., Treatment of metastatic melanoma with autologous CD4+ T cells against NY-ESO-1. N Engl J Med 2008, 358(25): 2698-703.

15. Stewart, T.J. and S.I. Abrams, How tumours escape mass destruction. Oncogene 2008, 27(45): 5894-903.

16. Banchereau, J., et al., Dendritic cells as melanoma vaccines. Dev Biol (Basel) 2004, 116: p. 14756; discussion 179-86.

17. Chhabra, A., N.G. Chakraborty, and B. Mukherji, Silencing of endogenous IL-10 in human dendritic cells leads to the generation of an improved CTL response against human melanoma associated antigenic epitope, MART-1 27-35. Clin Immunol 2008, 126(3): 251-9.

18. Gilboa, E. and J. Vieweg, Cancer immunotherapy with mRNA-transfected dendritic cells. Immunol Rev 2004, 199: 251-63. 
19. Chhabra, A., et al., Cross-presentation of a human tumor antigen delivered to dendritic cells by HSV VP22-mediated protein translocation. Eur J Immunol 2004, 34(10): 2824-33

20. Yuan, J., et al., CTLA-4 blockade enhances polyfunctional NY-ESO-1 specific T cell responses in metastatic melanoma patients with clinical benefit. Proc Natl Acad Sci U S 2008, 105(51): 20410-5.

21. Chhabra, A., et al., Activation-induced cell death of human melanoma specific cytotoxic $\mathrm{T}$ lymphocytes is mediated by apoptosis-inducing factor. Eur J Immunol 2006, 36(12): 3167-74.

22. Chhabra, A., Mitochondria-centric activation induced cell death of cytolytic T lymphocytes and its implications for cancer immunotherapy. Vaccine 2010, 28(29): 4566-72.

23. Eshhar, Z., et al., The T-body approach: potential for cancer immunotherapy. Springer seminars in immunopathology 1996, 18(2): 199-209.

24. Chhabra, A., TCR-engineered, customized, antitumor $\mathrm{T}$ cells for cancer immunotherapy: advantages and limitations. TheScientificWorldJournal 2011, 11: 121-9.

25. Sadelain, M., R. Brentjens, and I. Riviere, The promise and potential pitfalls of chimeric antigen receptors. Curr Opin Immunol 2009, 21(2): 215-23.

26. Thomson, J.A., et al., Embryonic stem cell lines derived from human blastocysts. Science 1998, 282(5391): 1145-7.

27. Wilmut, I., et al., Somatic cell nuclear transfer. Nature 2002, 419(6907): 583-6.

28. Surani, M.A., Nuclear reprogramming by human embryonic stem cells. Cell 2005, 122(5): 653-4.

29. Takahashi, K., et al., Induction of pluripotent stem cells from adult human fibroblasts by defined factors. Cell 2007, 131(5): 861-72.

30. Yu, J., et al., Induced pluripotent stem cell lines derived from human somatic cells. Science 2007, 318(5858): 1917-20.

31. Patel, M. and S. Yang, Advances in reprogramming somatic cells to induced pluripotent stem cells. Stem cell reviews 2010, 6(3): 367-80.

32. Tsuta, K., et al., Utility of 10 Immunohistochemical Markers Including Novel Markers (Desmocollin-3, Glypican 3, S100A2, S100A7, and Sox-2) for Differential Diagnosis of Squamous Cell Carcinoma from Adenocarcinoma of the Lung. Journal of thoracic oncology: official publication of the International Association for the Study of Lung Cancer 2011.

33. Gazouli, M., et al., OCT4 spliced variant OCT4B1 is expressed in human colorectal cancer. Molecular carcinogenesis 2011.

34. Liu, S.V., iPS cells: a more critical review. Stem cells and development 2008, 17(3): 391-7.

35. Kondo, M., et al., Biology of hematopoietic stem cells and progenitors: implications for clinical application. Annu Rev Immunol 2003, 21: 759-806.

36. Lansdorp, P.M., W. Dragowska, and H. Mayani, Ontogeny-related changes in proliferative potential of human hematopoietic cells. J Exp Med 1993, 178(3): 787-91.

37. Krause, D.S., et al., CD34: structure, biology, and clinical utility. Blood 1996, 87(1): 1-13.

38. DiGiusto, D., et al., Human fetal bone marrow early progenitors for T, B, and myeloid cells are found exclusively in the population expressing high levels of CD34. Blood 1994, 84(2): 421-32.

39. Slukvin, II, et al., Directed differentiation of human embryonic stem cells into functional dendritic cells through the myeloid pathway. J Immunol 2006, 176(5): 2924-32. 
40. Schmitt, T.M. and J.C. Zuniga-Pflucker, T-cell development, doing it in a dish. Immunol Rev 2006, 209: 95-102.

41. Schmitt, T.M. and J.C. Zuniga-Pflucker, Induction of $\mathrm{T}$ cell development from hematopoietic progenitor cells by delta-like-1 in vitro. Immunity 2002, 17(6): 749-56.

42. Zuniga-Pflucker, J.C., T-cell development made simple. Nat Rev Immunol 2004, 4(1): 67-72.

43. Choi, K.D., et al., Hematopoietic and endothelial differentiation of human induced pluripotent stem cells. Stem Cells 2009, 27(3): 559-67.

44. Kaufman, D.S., et al., Hematopoietic colony-forming cells derived from human embryonic stem cells. Proc Natl Acad Sci U S A 2001, 98(19): 10716-21.

45. Galic, Z., et al., T lineage differentiation from human embryonic stem cells. Proc Natl Acad Sci U $S$ A 2006, 103(31): 11742-7.

46. Orkin, S.H. and L.I. Zon, Hematopoiesis and stem cells: plasticity versus developmental heterogeneity. Nat Immunol 2002, 3(4): 323-8.

47. Hill, K.L. and D.S. Kaufman, Hematopoietic differentiation of human embryonic stem cells by cocultivation with stromal layers. Curr Protoc Stem Cell Biol 2008, Chapter 1: p. Unit 1F 6.

48. Wang, L., et al., Hematopoietic development from human embryonic stem cell lines. Exp Hematol 2005, 33(9): 987-96.

49. Vodyanik, M.A., et al., Human embryonic stem cell-derived CD34+ cells: efficient production in the coculture with OP9 stromal cells and analysis of lymphohematopoietic potential. Blood 2005, 105(2): 617-26.

50. Bandi, S. and R. Akkina, Human embryonic stem cell (hES) derived dendritic cells are functionally normal and are susceptible to HIV-1 infection. AIDS Res Ther 2008, 5: 1.

51. de Pooter, R.F. and J.C. Zuniga-Pflucker, Generation of immunocompetent $\mathrm{T}$ cells from embryonic stem cells. Methods Mol Biol 2007, 380: 73-82.

52. Novelli, E.M., M. Ramirez, and C.I. Civin, Biology of CD34+CD38- cells in lymphohematopoiesis. Leuk Lymphoma 1998, 31(3-4): 285-93.

53. Guo, Y., M. Lubbert, and M. Engelhardt, CD34- hematopoietic stem cells: current concepts and controversies. Stem Cells 2003, 21(1): 15-20.

54. Tseng, S.Y., et al., Generation of immunogenic dendritic cells from human embryonic stem cells without serum and feeder cells. Regenerative medicine, 2009, 4(4): 513-26.

55. Su, Z., et al., Differentiation of human embryonic stem cells into immunostimulatory dendritic cells under feeder-free culture conditions. Clinical cancer research: an official journal of the American Association for Cancer Research 2008, 14(19): 6207-17.

56. Senju, S., et al., Genetically manipulated human embryonic stem cell-derived dendritic cells with immune regulatory function. Stem cells 2007, 25(11): 2720-9.

57. Zhan, X., et al., Functional antigen-presenting leucocytes derived from human embryonic stem cells in vitro. Lancet 2004, 364(9429): 163-71.

58. Ray, S., et al., MHC-I-restricted melanoma antigen specific TCR-engineered human CD4+ T cells exhibit multifunctional effector and helper responses, in vitro. Clinical immunology 2010, 136(3): $338-47$. 
59. Chhabra, A., et al., CD4+CD25- T Cells Transduced to Express MHC Class I-Restricted EpitopeSpecific TCR Synthesize Th1 Cytokines and Exhibit MHC Class I-Restricted Cytolytic Effector Function in a Human Melanoma Model. J Immunol 2008, 181(2): 1063-70.

60. Bendle, G.M., et al., Lethal graft-versus-host disease in mouse models of $\mathrm{T}$ cell receptor gene therapy. Nature medicine 2010, 16(5): 565-70, 1p following 570.

61. Wrzesinski, C., et al., Hematopoietic stem cells promote the expansion and function of adoptively transferred antitumor CD8 T cells. The Journal of clinical investigation 2007, 117(2): 492-501.

62. Li, Y., et al., Vaccination with human pluripotent stem cells generates a broad spectrum of immunological and clinical responses against colon cancer. Stem cells 2009, 27(12): 3103-11.

63. Brewer, B.G., et al., Embryonic vaccines against cancer: an early history. Experimental and molecular pathology 2009, 86(3): 192-7.

64. Matsunaga, Y., et al., Activation of antigen-specific cytotoxic T lymphocytes by beta 2microglobulin or TAP1 gene disruption and the introduction of recipient-matched MHC class I gene in allogeneic embryonic stem cell-derived dendritic cells. Journal of immunology 2008, 181(9): 6635-43.

65. Senju, S., et al., Characterization of dendritic cells and macrophages generated by directed differentiation from mouse induced pluripotent stem cells. Stem cells 2009, 27(5): 1021-31.

(C) 2011 by the authors; licensee Insciences Journal.

Open Access article under the terms and conditions of Creative Commons Attribution Non-Commercial License 3.0 Unported. 